\title{
LCA in Green Chemistry: a new subject area and call for papers
}

\author{
Ivan Muñoz
}

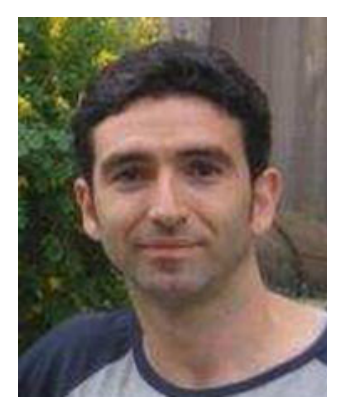

Received: 2 March 2012 / Accepted: 5 March 2012 /Published online: 17 April 2012

(C) Springer-Verlag 2012

\section{Introduction}

Chemicals surround us in all our daily activities. There is practically no facet in material life - transportation, communication, clothing, shelter, and office - in which chemistry does not play an important role, by supplying either consumer products or services. The development of the chemical industry in the past century resulted in huge advances, such as the development of effective drugs to cure diseases or the production of plant protection products and fertilizers that have increased the world's food supply. In spite of this, chemistry and its industry is often viewed by the general public as causing more harm than good (Lancaster 2002). Indeed, the manufacture, use, and disposal of chemicals consume large amounts of resources and originate emissions of pollutants to all environmental compartments. Given that the global demand for chemicals is expected to increase faster than the world's population and GDP (OECD 2001), there is a need for a shift towards a more efficient and sustainable chemistry.

The concept of Green Chemistry (GC) was coined by the US Environmental Protection Agency (USEPA) in the early 1990s and can be briefly defined as the use of chemistry for pollution prevention. Anastas et al. (2000) later defined it as "the design of chemical products and processes that reduce or eliminate the use and generation of hazardous substances." The term "hazard" in this definition was meant to include the full range of threats to human health and the environment, such as physical hazards, toxicity, climate change, and resource depletion (Anastas and Lankey

I. Muñoz $(\bowtie)$

Safety and Environmental Assurance Centre, Unilever,

Sharnbrook MK44 1LQ, UK

e-mail: ivan.munoz@unilever.com
2000). In order to make this concept operational, the USEPA developed a set of 12 guiding principles (Table 1). These principles constitute the backbone of GC and a universal code of practice for the eco-design of chemicals and chemical processes.

\section{The role of LCA in Green Chemistry}

A criticism to the 12 principles of $\mathrm{GC}$ as a tool is their qualitative nature, which can lead to difficulties when the progress made by introducing GC practices has to be measured, or when trade-offs between principles arise (Muñoz et al. 2006). The scientific community noticed this limitation, and several methods for environmental assessment in GC have been proposed (see Muñoz et al. 2006, Chap. 2). Life cycle assessment (LCA) has been highlighted as a suitable tool in this context by many authors (Lankey and Anastas 2002; Anastas and Lankey 2000; Domènech et al. 2002; Domènech 2005; Yasui 2003; Graedel 1999; Hellweg et al. 2004; Sikdar 2003). The potential role of LCA in GC can be summarized by quoting Gustafsson and Börjesson (2007), who concluded in their study on surface coatings about “... the importance of investigating the environmental performance of a product from cradle-to-grave and not only consider it 'green' because it is based on renewable resources or is produced based on the principles of Green Chemistry."

LCA is a well-known tool by the chemical industry, which uses it for several purposes, such as product and process development, marketing, and communication. Several companies apply it to their products, and their research has been often published in this journal (Saling et al. 2002; Curzons et al. 2007; Bretz and Frankhauser 1996). Nevertheless, several barriers and limitations regarding the use of LCA for GC exist, although they are not fundamentally 
Table 1 The 12 guiding principles of Green Chemistry

1. Prevention: It is better to prevent waste than to treat or clean up waste after it has been created

2. Atom economy: Synthetic methods should be designed to maximize the incorporation of all materials used in the process into the final product

3. Less hazardous chemical syntheses: Wherever practicable, synthetic methods should be designed to use and generate substances that possess little or no toxicity to human health and the environment

4. Designing safer chemicals: Chemical products should be designed to effect their desired function while minimizing their toxicity

5. Safer solvents and auxiliaries: The use of auxiliary substances (e.g., solvents, separation agents, etc.) should be made unnecessary wherever possible and innocuous when used

6. Design for energy efficiency: Energy requirements of chemical processes should be recognized for their environmental and economic impacts and should be minimized. If possible, synthetic methods should be conducted at ambient temperature and pressure

7. Use of renewable feedstocks: A raw material or feedstock should be renewable rather than depleting whenever technically and economically practicable

8. Reduce derivatives: Unnecessary derivatization (use of blocking groups, protection/deprotection, and temporary modification of physical/chemical processes) should be minimized or avoided if possible because such steps require additional reagents and can generate waste

9. Catalysis: Catalytic reagents (as selective as possible) are superior to stoichiometric reagents

10. Design for degradation: Chemical products should be designed so that at the end of their function they break down into innocuous degradation products and do not persist in the environment

11. Real-time analysis for pollution prevention: Analytical methodologies need to be further developed to allow for real-time, in-process monitoring and control prior to the formation of hazardous substances

12. Inherently safer chemistry for accident prevention: Substances and the form of a substance used in a chemical process should be chosen to minimize the potential for chemical accidents, including releases, explosions, and fires

different to those mentioned in other fields. These barriers include LCA being perceived as time-consuming and costly (Lancaster 2002), the lack of background inventory data for many chemicals (Wernet et al. 2009; Hischier et al. 2005), the lack of foreground data during the early stages of product development (Heinzle et al. 1998), and the focus on "potential" impacts rather than "actual" impacts or risks (accidents, exceeding toxicity thresholds in the environment, etc.) (Askham 2012). Although LCA cannot answer all the relevant questions on chemicals and their environmental impacts, it is my opinion that this is the best tool available as far as a holistic approach to the environmental impact of chemicals is concerned. This new section on GC and LCA in the International Journal of Life Cycle Assessment will be an excellent platform to prove it.

\section{Call for papers}

The publisher-editor and the subject editor invite submissions in the area of LCA in GC. These articles could include, though they are not limited to:

- Using LCA to measure the relative "greenness" of existing chemicals, chemical-containing products, and chemical processes: innovative case studies comparing different alternatives, and/or identifying for a single product or process where in the life cycle the impacts occur, and how to use LCA to define improvement strategies.

- Using LCA for screening the potential impacts of chemicals, chemical-containing products, and chemical processes under development: in this case, we refer to case studies using LCA in the early design stages, when data gaps are major, but so are the opportunities for environmental improvement.

- Methods to adapt LCA in order to assess chemicals or chemical processes in the early planning and design stage or when only bench-scale data are available: in relation to the above, how LCA practitioners should deal with the inherent uncertainty and lack of data they face when assessing a chemical product or process that only exists in a blueprint? Can we use LCA to make decisions at that stage?

- Approaches and models for bridging data gaps for chemicals: thousands of chemical substances may be involved in GC practices, whereas inventory data are not easily available (Hischier et al. 2005). Models to predict impacts from chemicals' production, based on molecular structure or other chemical properties, could be very useful tools for LCA application in GC (see Wernet et al. 2009).

- Tool comparisons, e.g., LCA vs. other assessment methods or metrics available for chemicals: when is it a good idea to use LCA, and when are other approaches better suited?

- Integration of LCA with other tools for an integrated assessment of chemicals: can LCA be used in a multicriteria assessment, along with other tools currently used for chemicals, like risk assessment? How can we accommodate potentially conflicting outcomes from these tools?

- Using LCA to identify potential benefits and trade-offs of applying the GC principles: do GC principles actually lead to improvements that can be measured with LCA? What principles should chemists and chemical engineers focus on in which cases?

- Reviews of LCA for the assessment of particular chemicals or chemical processes and on LCA as a tool for GC: providing a critical overview of literature in a particular area, identifying gaps and research needs. 


\section{References}

Anastas PT, Lankey RL (2000) Life cycle assessment and green chemistry: the ying and yang of industrial ecology. Green Chem 2:289-295

Anastas PT, Heine LG, Williamson TC (2000) Green chemical syntheses and processes: introduction. In: Anastas PT, Heine LG, Williamson TC (eds) Green chemical syntheses and processes. American Chemical Society, Washington DC

Askham C (2012) REACH and LCA - methodological approaches and challenges. Int J Life Cycle Assess 17:43-57

Bretz R, Frankhauser P (1996) Screening LCA for large numbers of products. Estimation tools to fill data gaps. Int J Life Cycle Assess 1(3):139-146

Curzons AD, Jiménez-González C, Duncan AL, Constable DJC, Cunningham VL (2007) Fast life cycle assessment of synthetic chemistry (FLASC ${ }^{\mathrm{TM}}$ ) tool. Int J Life Cycle Assess 12(4):272-280

Domènech X (2005) Química Verde. Rubes Editorial, Barcelona

Domènech X, Ayllón JA, Peral J, Rieradevall J (2002) How green is a chemical reaction? Application of LCA to green chemistry. Environ Sci Technol 36:5517-5520

Graedel T (1999) Green chemistry in an industrial ecology context. Green Chem 1(5):G126-G128

Gustafsson LM, Börjesson P (2007) Life cycle assessment in green chemistry. A comparison of various industrial wood surface coatings. Int J Life Cycle Assess 12(3):151-159

Heinzle E, Weirich D, Brogli F, Hoffmann VH, Koller G, Verduyn MA, Hungerbühler K (1998) Ecological and economic objective functions for screening in integrated development of fine chemical processes. 1. Flexible and expandable framework using indices. Ind Eng Chem Res 37:3395-3407
Hellweg S, Fischer U, Scheringer M, Hungerbühler K (2004) Environmental assessment of chemicals: methods and application to a case study of organic solvents. Green Chem 6:418-427

Hischier R, Hellweg S, Capello C, Primas A (2005) Establishing life cycle inventories of chemicals based on differing data availability. Int J Life Cycle Assess 10(1):59-67

Lancaster M (2002) Green chemistry: an introductory text. RSC Paperbacks, UK

Lankey RL, Anastas PT (2002) Life-cycle approaches for assessing green chemistry technologies. Ind Eng Chem Res 41:4498-4502

Muñoz I, Domènech X, Malato S (2006) Life cycle assessment as a tool for green chemistry: application to different advanced oxidation processes for wastewater treatment. Centro de Investigaciones Energéticas, medioambientales y Tecnológicas, Madrid. ISBN 84-7834-520-5. The $\mathrm{PhD}$ thesis version of this book is available at: http://hdl.handle.net/10803/3223. Accessed 2 Oct 2012

OECD (2001) Environmental outlook for the chemicals industry. OECD Environment Directorate, Paris

Saling P, Kicherer A, Dittrich-Krämer B, Wittlinger R, Zombik W, Schmidt I, Schrott W, Schmidt S (2002) Eco-efficiency by BASF: the method. Int J Life Cycle Assess 7(4):203-218

Sikdar SK (2003) Sustainable development and sustainability metrics. AICHE J 49(8):1928-1932

Wernet G, Papadokonstantakis S, Hellweg S, Hungerbuhler K (2009) Bridging data gaps in environmental assessments: modeling impacts of fine and basic chemical production. Green Chem 11:1826-1831

Yasui I (2003) Metrics for green and sustainable chemistry. Green Chem 5(5):G70-G73 